\title{
Dravet syndrome
}

INSERM

\section{Source}

INSERM. (1999). Orphanet: an online rare disease and orphan drug data base. Dravet syndrome. ORPHA:33069

Dravet syndrome (DS) is a genetic epilepsy of childhood characterized by a variety of drug-resistant seizures often induced by fever, presenting in previously healthy children, and which frequently leads to cognitive and motor impairment. 